\title{
Computed tomography coronary angiography accuracy in women and men at low to intermediate risk of coronary artery disease
}

\author{
Anoeshka S. Dharampal • Stella L. Papadopoulou • \\ Alexia Rossi • Annick C. Weustink • Nico R. A. Mollet • \\ W. Bob Meijboom • Lisan A. Neefjes • Koen Nieman • \\ Eric Boersma • Pim J. de Feijter • Gabriel P. Krestin
}

Received: 6 January 2012 /Revised: 23 March 2012 / Accepted: 12 April 2012 / Published online: 6 June 2012

(C) The Author(s) 2012. This article is published with open access at Springerlink.com

\begin{abstract}
Objectives To investigate the diagnostic accuracy of CT coronary angiography (CTCA) in women at low to intermediate pre-test probability of coronary artery disease (CAD) compared with men.

Methods In this retrospective study we included symptomatic patients with low to intermediate risk who underwent both invasive coronary angiography and CTCA. Exclusion criteria were previous revascularisation or myocardial infarction. The pre-test probability of CAD was estimated using the Duke risk score. Thresholds of less than $30 \%$ and 30-90\% were used for determining low and intermediate risk, respectively. The diagnostic accuracy of CTCA in detecting obstructive CAD ( $\geq 50 \%$ lumen diameter narrowing) was calculated on patient level. $P<0.05$ was considered significant.

Results A total of 570 patients (46\% women [262/570]) were included and stratified as low (women $73 \%$ [80/109]) and intermediate risk (women $39 \%$ [182/461]). Sensitivity, specificity, PPV and NPV were not significantly different in and between women and men at low and intermediate risk. For women vs. men at low risk they were $97 \%$ vs. $100 \%$,
\end{abstract}

A. S. Dharampal $(\bowtie) \cdot$ S. L. Papadopoulou · A. Rossi

A. C. Weustink $\cdot$ N. R. A. Mollet $\cdot$ W. B. Meijboom •

L. A. Neefjes $\cdot$ K. Nieman · P. J. de Feijter · G. P. Krestin

Department of Radiology, Erasmus MC,

Room Ca207a, 's-Gravendijkwal 230, P.O. Box 2040, 3015 CE,

Rotterdam, The Netherlands

e-mail: a.dharampal@erasmusmc.nl

A. S. Dharampal · S. L. Papadopoulou $\cdot$ A. Rossi

A. C. Weustink $\cdot$ N. R. A. Mollet $\cdot$ W. B. Meijboom •

L. A. Neefjes $\cdot$ K. Nieman $\cdot$ E. Boersma $\cdot$ P. J. de Feijter

Department of Cardiology, Erasmus MC,

Rotterdam, The Netherlands
$79 \%$ vs. $90 \%, 80 \%$ vs. $80 \%$ and $97 \%$ vs. $100 \%$, respectively. For intermediate risk they were $99 \%$ vs. $99 \%, 72 \%$ vs. $83 \%, 88 \%$ vs. $93 \%$ and $98 \%$ vs. $99 \%$, respectively.

Conclusion CTCA has similar diagnostic accuracy in women and men at low and intermediate risk.

Key Points

- Coronary artery disease (CAD) is increasingly investigated by computed tomography angiography (CTCA).

- CAD detection or exclusion by CTCA is not different between sexes.

- CTCA diagnostic accuracy was similar between low and intermediate risk sex-specific-groups.

- CTCA rarely misses obstructive CAD in low-intermediate risk women and men.

- CAD yield by invasive coronary angiography after positive CTCA is similar between sex-risk-specific groups.

Keywords Diagnostic accuracy · CT coronary angiography · Multidetector computed tomography $\cdot$ Coronary artery disease $\cdot$ Duke pre-test probability $\cdot$ Sex, women and men

$\begin{array}{ll}\text { Abbreviations } \\ \text { AUC } & \text { area under receiver-operating characteristic curve } \\ \text { CAD } & \text { coronary artery disease } \\ \text { CTCA } & \text { computed tomography coronary angiography } \\ \text { ECG } & \text { electrocardiography } \\ \text { HR } & \text { heart rate } \\ \text { ICA } & \text { invasive coronary angiography } \\ \text { NPV } & \text { negative predictive value } \\ \text { PPV } & \text { positive predictive value } \\ \text { PTP } & \text { pre-test probability } \\ \text { SN } & \text { sensitivity }\end{array}$




\section{SP specificity}

SPECT single photon emission computed tomography

QCA quantitative coronary angiography

\section{Introduction}

Cardiovascular disease is the leading cause of death in the western world [1]. For several years awareness has been growing that cardiovascular disease is also the primary cause of death in women not only in the western world but also in economic developing countries [2]. Part of this high mortality is related to under-recognition, underdiagnosis and undertreatment of coronary artery disease (CAD) in women [3-7].

The interpretation of chest pain in women caused by CAD is often difficult because of the "less typical" symptoms in women compared with the classical "typical" symptoms in men $[3,4,8]$. This may cause a delay or lead to incorrect diagnosis [3, 4]. Additionally first-line non-invasive diagnostic tests such as exercise electrocardiography and single photon emission computed tomography (SPECT) imaging in women are less sensitive and specific $[5,6]$.

Paradoxically, because of the diagnostic uncertainty there appears to be an overuse of invasive coronary angiography (ICA) in women, with a rather low diagnostic yield of obstructive CAD $[9,10]$. This prompted a "red alert call" for the promotion of advanced non-invasive imaging techniques in women including CT coronary angiography (CTCA) [11]. CTCA has evolved as a reliable gatekeeper of ICA because negative CT findings virtually rule out the presence of obstructive CAD [12-14]. Whether this also applies to women remains unresolved because women have been under-represented in studies assessing the diagnostic accuracy of CTCA [12-17]. The pre-test probability (PTP) of obstructive CAD plays a significant role in the assessment of the clinical utility of CTCA. This PTP of obstructive $\mathrm{CAD}$ can be derived from reported clinical prediction rules [18] and may influence the post-CTCA probability of obstructive $\mathrm{CAD}$ and thus the need for further testing and management.

The purpose of our study was to assess the diagnostic accuracy and clinical utility of CTCA to detect or exclude obstructive CAD in women with low to intermediate PTP of obstructive $\mathrm{CAD}$ in comparison to men.

\section{Methods and materials}

Study population

Patients referred by their treating physician for ICA between July 2004 and June 2009 on the basis of chest pain presentation (typical, atypical and non-anginal complaints) with or without outcome of stress testing were invited to undergo CTCA within 2 weeks of ICA. The CTCA outcome did not affect referral to ICA. We excluded patients with known iodine allergy, impaired renal function and patients with previous revascularisation. A total of 907 patients gave consent for CT accuracy studies and were entered in our single-centre registry. Out of these 907 patients we excluded patients with previous myocardial infarct $(n=54)$ as well as patients with a high PTP ( $>90 \%)$ of having obstructive CAD $(n=283)$. The final study sample comprised a total of 570 symptomatic patients. The study was approved by the institutional review board of our medical hospital.

Computed tomography imaging protocol and image reconstruction

Patients underwent single-source CT (Somatom Sensation, Siemens Healthcare, Forchheim, Germany) between 2004 and 2006 and subsequently underwent dual-source CT (Somatom Definition, Siemens Healthcare). The imaging settings are described in Table 1. Heart-rate-lowering medication (Metoprolol, Seloken, Astra Zeneca, Zoetermeer, Netherlands) was administered when the heart rate was above 65 beats per minute before the single-source $\mathrm{CT}$ in the absence of contraindications to achieve better quality images. To achieve better visualisation of the small coronary arteries the vasodilating agent Nitrolingual (Nitroglycerin Pumpspray, G.Pohl-boskamp, Itohenlockstedt, Germany) was administered before imaging in the absence of contraindications.

All patients initially underwent unenhanced CT to calculate the calcium score using the Agatston method [19]. Subsequently a bolus tracking technique was used to synchronise the start of image acquisition with the arrival of the iodinated contrast agent [Iomeprol, iomeron (400 milligram iodine per millilitre), Bracco, Milan, Italy; Ultravist, iopromide (370 milligram iodine per millilitre), Schering Berlin, Germany] in the coronary arteries followed by a saline chaser.

Between 2004 and 2006 a full-dose ECG-synchronised spiral imaging technique was used. After the introduction of ECG-triggered tube current modulation, the spiral imaging technique with ECG pulsing was used in the subsequent years to reduce the effective radiation dose. Data sets were reconstructed retrospectively according to the heart rate to obtain motion-free images. For heart rates of at least 80 beats per minute the data sets were reconstructed in 31$47 \%$ of the R-R interval (systolic phases). For heart rates of no greater than 65 beats per minute this value was $60-76 \%$ of the R-R interval (diastolic phases). For heart rates between 66 and 79 beats per minute reconstructions were needed in both systolic and diastolic phases, 30-77\% of the $\mathrm{R}-\mathrm{R}$ interval. Images were analysed using medium-to- 
Table 1 Imaging protocol

\begin{tabular}{|c|c|c|}
\hline & $\mathrm{SSCT}^{\mathrm{b}}$ & $\mathrm{DSCT}^{\mathrm{c}}$ \\
\hline$\beta$-blocker ${ }^{\mathrm{a}}$ & Yes & No \\
\hline Nitroglycerin $^{\mathrm{a}}$ & No & Yes \\
\hline X-ray tube & 1 & 2 \\
\hline Collimation & $32 \times 0.6(\mathrm{Z}-\mathrm{FFS})$ & $32 \times 0.6(\mathrm{Z}-\mathrm{FFS})$ \\
\hline Gantry rotation time $(\mathrm{ms})$ & 330 & 330 \\
\hline Temporal resolution $(\mathrm{ms})$ & 165 & 83 \\
\hline Spatial resolution $(\mathrm{mm})$ & $0.4 \times 0.4 \times 0.4$ & $0.4 \times 0.4 \times 0.4$ \\
\hline Pitch & 0.2 & $0.2-0.53$ \\
\hline Rotation time (ms) & 330 & 330 \\
\hline \multicolumn{3}{|l|}{ Unenhanced imaging } \\
\hline Tube voltage $(\mathrm{kV})$ & 120 & 120 \\
\hline Tube current & $\begin{array}{l}200-150 \text { effective } \\
\text { mAs }\end{array}$ & $75 \mathrm{mAs} /$ rotation \\
\hline $\begin{array}{l}\text { ECG-triggered tube current } \\
\text { modulation } \\
\text { Contrast-enhanced imaging }\end{array}$ & Yes & Yes \\
\hline Tube voltage $(\mathrm{kV})$ & 120 & 120 \\
\hline Tube current & $\begin{array}{l}850-960 \\
\text { effective mAs }\end{array}$ & $\begin{array}{l}320-412 \mathrm{mAs} / \\
\text { rotation }\end{array}$ \\
\hline $\begin{array}{l}\text { ECG-triggered tube current } \\
\text { modulation }\end{array}$ & No & Yes \\
\hline
\end{tabular}

SSCT single-source CT, DSCT dual-source CT, Z-FFS Z-flying focal spot, $E C G$ electrocardiogram, $m A$ s/rotation total $\mathrm{mA} \times$ rotation time

${ }^{a}$ Administration before imaging when no contraindication was present

${ }^{\mathrm{b}}$ Somatom Sensation, Siemens Healthcare, Forchheim, Germany

${ }^{\mathrm{c}}$ Somatom Definition, Siemens Healthcare, Forchheim, Germany

smooth convolution kernels for non-calcified lesions and sharp convolution kernels for calcified lesions.

\section{Computed tomography image evaluation}

All data sets were transferred for analyses to an offline proprietary workstation (Leonardo, Siemens, Erlangen, Germany); the total calcium score was calculated using dedicated proprietary software (CaScoring). Two experienced observers with more than 2 years of experience in cardiac $\mathrm{CT}$, blinded to the ICA results, independently evaluated all CTCAs for the presence of CAD, using axial source images, multiplanar, curved reformatted reconstructions, and thinslab maximum intensity projections using "Circulation" software on the proprietary workstation. Interobserver disagreements were resolved by a joint consensus reading.

The modified 17-segment American Heart Association model was used to classify each segment [20]. Each segment was visually scored as obstructive in the presence of at least $50 \%$ lumen diameter narrowing and non-obstructive when the lumen diameter narrowing was less than $50 \%$ in comparison with the proximal and distal lumen. All anatomically available coronary segments with a diameter of at least $1.5 \mathrm{~mm}$, irrespective of image quality or calcification, were included and scored with the intention to diagnose. Un-evaluable segments of poor quality due to calcification, stack, motion artefacts, or low contrast enhancement were scored as obstructive. Coronary segments distal to a total occlusion could not be optimally visualised by ICA and thus were excluded from the analyses. The image quality was scored on a segment level as diagnostic and non-diagnostic. The average of this score per patient was compared between women and men.

Invasive coronary angiogram image evaluation

One experienced cardiologist, blinded to the CT results, visually assessed each coronary segment (American Heart Association model) for the presence of luminal narrowing in two orthogonal planes. Stenoses scored as having more than $20 \%$ narrowing on visual assessment were quantified using the validated quantitative coronary angiography (QCA) algorithm (CAASII [Cardiovascular Angiography Analysis System II]; Pie Medical Imaging Maastricht, the Netherlands). The segments were considered obstructive when the quantified lumen diameter narrowing in one of the two planes was at least $50 \%$. Obstructive lesions on a segment level were used to determine obstructive CAD on a patient level as having one or more obstructive stenoses $(\geq 50 \%$ lumen diameter stenosis) irrespective of the segment in which they were located.

\section{PTP of obstructive CAD}

We used the Duke risk score [18] to estimate the PTP of obstructive CAD due to the presence of multiple risk factors such as age, gender, symptoms, history of myocardial infarction, ECG, smoking, hypercholesterolaemia and diabetes mellitus. We used less than $30 \%, 30-90 \%$, and greater than $90 \%$ as thresholds for the low, intermediate and high risk group, respectively. As patients with a high risk are directly referred for ICA we did not include these patients in our analyses [21, 22].

The observed PTP of obstructive CAD is the prevalence of obstructive CAD defined as having at least one lesion with at least $50 \%$ lumen diameter stenosis per patient detected by ICA.

\section{Statistical analyses}

The statistical analysis was performed using a dedicated statistical software program (SPSS, version 16.0, IMB, Chicago, IL, USA). Categorical variables were expressed as percentages and continuous variables were expressed as means \pm standard deviation. Continuous variables with a 
skewed distribution were expressed as median with interquartile range.

The estimated PTP was compared in women, men and in the different risk groups with the observed PTP (observed prevalence) using the paired $t$ test.

The patients' characteristics were compared between women and men and between different risk groups using the independent $t$ test; otherwise the Mann-Whitney $U$ test was used (skewed distribution of continuous data). The interobserver variability was tested between the two CT readers on segment and patient level using the $\kappa$ statistic.

The CTCA results were compared with the reference standard ICA on a patient level to calculate the sensitivity (SN), specificity (SP), positive predictive value (PPV) and negative predictive value (NPV) [23]. The Wilson score [24] was used to calculate the confidence intervals for small groups. The diagnostic accuracy and utility were compared across the different risk groups, between women and men using the chi-squared test or the Fisher's exact test in the presence of less than five observations in a cell of the $2 \times 2$ table. A $P$ value of less than 0.05 was considered statistically significant.

The receiver-operating characteristic (ROC) curve was used for visual analyses of the trade-offs between the sensitivity and the specificity of CTCA. The area under the ROC curve (AUC) was calculated to account for the influence of referral bias and prevalence of disease on the diagnostic accuracy of CTCA in our population $[25,26]$.

The clinical utility of CTCA was a measure of diagnostic certainty or remaining uncertainty of absence or presence of CAD that is derived from the post-test probability of CAD. In case of diagnostic certainty no further testing is required, whereas remaining uncertainty requires further diagnostic testing. It was assumed that a post-test probability of obstructive CAD of less than 5\% indicated high certainty with no further requirement for diagnostic testing; probabilities between 5 and $90 \%$ represented uncertainty and indicated a requirement for further diagnostic testing; and probabilities of greater than $90 \%$ indicated high certainty with direct referral to ICA, as proposed by previous studies [27].

This study was performed according to the criteria set forth in the Standard for Reporting of Diagnostic Accuracy Initiative [28].

\section{Results}

In total, 570 patients at low to intermediate risk were included of which $46 \%$ were women $(n=262)$. The patients' characteristics are provided according to sex and risk group in Table 2. Women overall had a significantly higher heart rate $(\mathrm{HR})$ than men $(P=0.03)$. The HR was similar in the different risk groups (Table 2). The prevalence of CAD (at least one obstructive lesion) in women was $61 \%$ and in men this was $64 \%(P=0.42)$. The PTP of obstructive CAD determined by the Duke risk score (estimated PTP) was significantly lower than the observed prevalence of obstructive CAD determined by ICA in women in the overall group ( $45 \%$ vs. $61 \%, P<0.01)$ as well as in the low $(17 \%$ vs. $48 \%, P<0.01)$ and intermediate risk groups $(60 \%$ vs. $66 \%$, $P=0.02)$ separately. In men there was no difference between the estimated PTP and observed prevalence (overall $65 \%$ vs. $64 \%, P=0.56$; low $21 \%$ vs. $28 \%, P=0.43$; intermediate risk group ( $70 \%$ vs. $68 \%, P=0.38$ ).

In this population the prevalence of obstructive $\mathrm{CAD}$ and extent of CAD in terms of multi-vessel disease and calcium score were similar between women and men (Table 2).

The interobserver variability for detection of obstructive stenosis showed a good agreement on segment $(\kappa=0.94$ [95\% CI 0.93-0.95]) and patient levels $(\kappa=0.91$ [95\% CI $0.87-$ 0.95]). The diagnostic image quality was not significantly different between women and men (0.96 vs. $0.97, P=0.06$ ).

\section{Diagnostic accuracy of CTCA}

The patient-based diagnostic accuracy of CTCA for detecting or ruling out obstructive $\mathrm{CAD}$ according to ICA revealed an AUC of 0.895 (95\% CI: $0.862-0.928)$ which was similar between women and men (0.867 [95\% CI: $0.815-0.920$ ] vs. 0.921 [95 \% CI: 0.880 - 0.961], $P=0.06$ ), with similar sensitivity and specificity for women and men when grouped in the low and intermediate risk groups and across the low and intermediate risk groups in both women and men (Table 3).

Because different CT systems were used over the years we also assessed the diagnostic performance for detecting CAD between these two machines. No significant differences were found between the single-source and dual-source CT systems for sensitivity ( $99 \%$ vs. $99 \%, P=0.93$ ), specificity ( $84 \%$ vs. $75 \%, P=0.10)$, positive predictive value ( $85 \%$ vs. $91 \%, P=$ 0.06 ) and negative predictive value ( $99 \%$ vs. $97 \%, P=0.39$ ).

\section{Clinical utility of CTCA}

Certainty defined as probabilities for obstructive CAD of no greater than $5 \%$ after negative CTCA was achieved in both women (NPV $97 \%$ and $98 \%$ for low and intermediate risk, respectively) and men (NPV $100 \%$ and $99 \%$ for low and intermediate risk, respectively) at low to intermediate risk. No certainty of obstructive CAD after positive CTCA could be achieved (probability $<90 \%$ ) in women at low (PPV $80 \%$ ) and intermediate risk (PPV $88 \%$ ), and men at low risk (PPV $80 \%$ ) (Table 3).

Yield of obstructive CAD by ICA after CTCA

A negative CTCA would prevent $31 \%(174 / 570)$ of the low to intermediate risk patients proceeding to ICA (30\% (79/262) 
Table 2 Patients' characteristics

\begin{tabular}{|c|c|c|c|c|c|c|c|c|c|c|c|c|}
\hline & \multicolumn{4}{|l|}{ All } & \multicolumn{4}{|l|}{ Low } & \multicolumn{4}{|l|}{ Intermediate } \\
\hline & \multicolumn{2}{|l|}{ Women } & \multicolumn{2}{|l|}{ Men } & \multicolumn{2}{|l|}{ Women } & \multicolumn{2}{|l|}{ Men } & \multicolumn{2}{|l|}{ Women } & \multicolumn{2}{|l|}{ Men } \\
\hline & Mean & {$[\mathrm{SD}]$} & Mean & {$[\mathrm{SD}]$} & Mean & {$[\mathrm{SD}]$} & Mean & {$[\mathrm{SD}]$} & Mean & {$[\mathrm{SD}]$} & Mean & {$[\mathrm{SD}]$} \\
\hline Number patients & 262 & & 308 & & 80 & & 29 & & 182 & & 279 & \\
\hline Age (years) & $62^{*}$ & {$[10.96]$} & $55^{*}$ & {$[9.52]$} & $54 *$ & {$[8.58]$} & $40^{*}$ & {$[8.15]$} & $66^{*}$ & {$[9.57]$} & $57^{*}$ & {$[8.18]$} \\
\hline \multicolumn{13}{|l|}{ Chest pain complaints ${ }^{\mathrm{a}}$} \\
\hline Typical & $30 \% *(78)$ & {$[0.46]$} & $20 \% *(63)$ & {$[0.40]$} & $1 \% *(1)$ & {$[0.11]$} & $0 \% *(0)$ & {$[0.00]$} & $42 \% *(77)$ & {$[0.50]$} & $23 \% *(63)$ & {$[0.42]$} \\
\hline Atypical & $38 \% *(100)$ & {$[0.49]$} & $50 \% *(155)$ & {$[0.50]$} & $49 \% *(39)$ & {$[0.50]$} & $41 \% *(12)$ & {$[0.50]$} & $34 \% *(61)$ & {$[0.47]$} & $51 \% *(143)$ & {$[0.50]$} \\
\hline Non-anginal & $32 \%(84)$ & {$[0.47]$} & $29 \%(90)$ & {$[0.46]$} & $50 \%(40)$ & {$[0.50]$} & $59 \%(17)$ & {$[0.50]$} & $24 \%(44)$ & {$[0.43]$} & $26 \%(73)$ & {$[0.44]$} \\
\hline Current smoker & $23 \%(59)$ & {$[0.42]$} & $28 \%(86)$ & {$[0.45]$} & $14 \%(11)$ & {$[0.34]$} & $10 \%(3)$ & {$[0.31]$} & $26 \%(48)$ & {$[0.44]$} & $29 \%(83)$ & {$[0.46]$} \\
\hline Diabetes mellitus ${ }^{\mathrm{b}}$ & $16 \%(43)$ & {$[0.37]$} & $12 \%(36)$ & {$[0.33]$} & $4 \%(3)$ & {$[0.19]$} & $0 \%(0)$ & {$[0.00]$} & $22 \% *(40)$ & {$[0.42]$} & $13 \% *(36)$ & {$[0.34]$} \\
\hline CAD in family ${ }^{\mathrm{c}}$ & $55 \% *(143)$ & {$[0.50]$} & $44 \% *(137)$ & {$[0.50]$} & $59 \%(47)$ & {$[0.50]$} & $41 \%(12)$ & {$[0.50]$} & $53 \%(96)$ & {$[0.50]$} & $45 \%(125)$ & {$[0.50]$} \\
\hline Hypercholesterolaemia $^{\mathrm{d}}$ & $56 \% *(147)$ & {$[0.50]$} & $44 \% *(135)$ & {$[0.50]$} & $41 \% *(35)$ & {$[0.50]$} & $10 \% *(3)$ & {$[0.31]$} & $62 \% *(112)$ & [0.49] & $47 \% *(132)$ & {$[0.50]$} \\
\hline Hypertension $^{\mathrm{e}}$ & $52 \% *(138)$ & {$[0.50]$} & $40 \% *(122)$ & {$[0.49]$} & $48 \% *(38)$ & {$[0.50]$} & $10 \% *(3)$ & {$[0.31]$} & $54 \% *(100)$ & {$[0.50]$} & $42 \% *(119)$ & {$[0.50]$} \\
\hline $\begin{array}{l}\text { Body mass index } \\
\left(\mathrm{kg} / \mathrm{m}^{2}\right)\end{array}$ & 27 & {$[4.45]$} & 27 & {$[3.51]$} & 27 & {$[4.62]$} & 26 & {$[2.56]$} & 27 & {$[4.38]$} & 27 & [3.59] \\
\hline Calcium score ${ }^{\mathrm{f}}$ & $139 *$ & {$[5-446]$} & $349^{*}$ & [9-401] & 20 & [0-194] & 2 & {$[0-65]$} & 211 & [36-613] & 155 & {$\left[\begin{array}{ll}14 & 444]\end{array}\right.$} \\
\hline $\begin{array}{l}\text { Prevalence CAD } \\
\text { segments/patient }\end{array}$ & $9 \%$ & {$[0.11]$} & $10 \%$ & {$[0.12]$} & $7 \% *$ & {$[0.09]$} & $3 \% *$ & {$[0.06]$} & $10 \%$ & {$[0.11]$} & $11 \%$ & {$[0.13]$} \\
\hline One vessel disease & $35 \%(92)$ & {$[0.48]$} & $32 \%(98)$ & {$[0.47]$} & $33 \%(26)$ & {$[0.47]$} & $21 \%(6)$ & {$[0.41]$} & $36 \%(66)$ & {$[0.48]$} & $33 \%(92)$ & {$[0.47]$} \\
\hline Multi-vessel disease $^{\mathrm{h}}$ & $25 \%(65)$ & {$[0.43]$} & $32 \%(99)$ & {$[0.47]$} & $14 \%(11)$ & {$[0.35]$} & $7 \%(2)$ & {$[0.26]$} & $30 \%(54)$ & {$[0.46]$} & $35 \%(97)$ & {$[0.48]$} \\
\hline Estimated $\mathrm{PTP}^{\mathrm{i}}$ & $45 \% *$ & {$[0.25]$} & $65 \% *$ & {$[0.21]$} & $17 \% *$ & {$[0.07]$} & $21 \% *$ & {$[0.08]$} & $60 \% *$ & {$[0.18]$} & $70 \% *$ & {$[0.17]$} \\
\hline Observed PTP & $61 \%(159)$ & {$[0.49]$} & $64 \%(197)$ & {$[0.48]$} & $48 \%(38)$ & {$[0.50]$} & $28 \%(8)$ & {$[0.45]$} & $66 \%(121)$ & {$[0.47]$} & $68 \%(189)$ & {$[0.47]$} \\
\hline HR (beats per minute) & $67^{*}$ & {$[11.94]$} & $65^{*}$ & {$[12.64]$} & 67 & [11.91] & 65 & [13.78] & 67 & [11.98] & 65 & [12.54] \\
\hline $\begin{array}{l}\text { Radiation exposure } \\
(\mathrm{mSv})^{\mathrm{k}}\end{array}$ & 12 & {$[3.78]$} & 12 & {$[4.05]$} & 12 & {$[3.24]$} & 13 & {$[3.56]$} & 12 & {$[3.92]$} & 12 & [4.09] \\
\hline
\end{tabular}

Data in parentheses are number of patients. Data in brackets are the standard deviations and for the calcium score the 25th and 75th percentiles *Bold values represent significant values $(P<0.05)$ in the comparison between women and men using Student's $t$ test or Mann-Whitney $U$ test (italics)

${ }^{a}$ We defined typical angina as substernal discomfort that was precipitated by physical exertion or emotion and relieved by rest or nitroglycerin within $10 \mathrm{~min}$. We classified chest pain with only 1 or 2 of these 3 symptom characteristics as atypical angina pectoris; if none of the characteristics was present, we classified it as non-anginal chest pain

${ }^{\mathrm{b}}$ Treatment with oral antidiabetic medication or insulin

${ }^{\mathrm{c}}$ Patient had first- or second-degree relatives with premature CAD

d Total cholesterol $>180 \mathrm{mg} / \mathrm{dl}$ or treatment for hypercholesterolaemia

${ }^{\mathrm{e}}$ Blood pressure $>120 / 90 \mathrm{mmHg}$ or treatment for hypertension

${ }^{\mathrm{f}}$ Agatston score with median and [interquartile range]

${ }^{\mathrm{g}}[($ Number diseased segments $\times 100 \%) /($ total of number segments)] per patient

$\mathrm{h}^{\mathrm{h}}>1$ vessel with obstructive CAD detected by ICA

${ }^{\mathrm{i}}$ Estimated pre-test probability of obstructive CAD [18]: low, <30 \% (estimated PTP); intermediate, 30-90 \% (estimated PTP)

${ }^{\mathrm{j}}$ Prevalence of obstructive CAD on a patient level: observed probability of obstructive CAD determined by ICA

${ }^{\mathrm{k}} \mathrm{mSv}$ (millisievert): dose length product $\times 0.017$

women; $31 \%(95 / 308)$ men, $P=0.86$; Fig. 1). This prevention of ICA would be more pronounced in patients at low risk (low $49 \%(53 / 109)$ vs. intermediate $26 \%(121 / 461), P<0.001)$ compared to patients at intermediate risk. Obstructive CAD would be missed in less than $1 \%$ after a negative CTCA with no significant differences between women and men (1\% (2/ $262)$ women vs. $0 \%(1 / 308)$ men, $P=0.48)$, or between the low and intermediate risk groups ( $1 \%(1 / 109)$ low vs. $0 \%(2 /$ 461 ) intermediate risk group, $P=0.52$ ). A positive CTCA would result in $89 \%$ (353/396) yield of obstructive CAD by
ICA with no significant differences between women and men ( $86 \%(157 / 183)$ women vs. $92 \%(196 / 213)$ men, $P=0.0502)$ or between the low and intermediate risk groups $(80 \%(45 / 56)$ low vs. $91 \%$ (308/340) intermediate risk group, $P=0.06)$.

\section{Discussion}

In our clinical evaluation of CTCA in low to intermediate risk women and men with suspected $\mathrm{CAD}$ who were 
Table 3 Diagnostic accuracy and clinical utility of CTCA between women and men across different risk groups

\begin{tabular}{|c|c|c|c|c|c|c|c|c|c|c|c|c|c|}
\hline \multirow[t]{2}{*}{ Risk groups } & & \multirow{2}{*}{$\begin{array}{l}\text { Women } \\
n\end{array}$} & \multicolumn{2}{|c|}{ Men } & \multicolumn{4}{|l|}{ Women } & \multicolumn{4}{|l|}{ Men } & \multirow{2}{*}{$\begin{array}{l}P \text { value } \\
(\mathrm{W}-\mathrm{M})\end{array}$} \\
\hline & & & $n$ & & $\mathrm{SN}$ & SP & PPV & NPV & SN & SP & PPV & NPV & \\
\hline \multirow[t]{4}{*}{ All } & $\mathrm{TP}$ & 157 & 196 & SN & 99 [96-100] & & & & 99 [97-100] & & & & $0.59 *$ \\
\hline & $\mathrm{TN}$ & 77 & 94 & SP & & 77 [66-82] & & & & 85 [77-90] & & & 0.07 \\
\hline & $\mathrm{FP}$ & 26 & 17 & PPV & & & $86[80-90]$ & & & & 92 [88-95] & & 0.047 \\
\hline & FN & 2 & 1 & NPV & & & & 97 [91-99] & & & & 99 [94-100] & $0.59^{*}$ \\
\hline \multirow[t]{4}{*}{ Low } & $\mathrm{TP}$ & 37 & 8 & SN & 97 [87-100] & & & & $100[68-100]$ & & & & $1.00 *$ \\
\hline & $\mathrm{TN}$ & 33 & 19 & SP & & 79 [64-88] & & & & 90 [71-97] & & & $0.31 *$ \\
\hline & FP & 9 & 2 & PPV & & & 80 [67-89] & & & & 80 [49-94] & & $1.00^{*}$ \\
\hline & $\mathrm{FN}$ & 1 & 0 & NPV & & & & 97 [85-99] & & & & $100[83-100]$ & $1.00 *$ \\
\hline \multirow[t]{4}{*}{ Intermediate } & $\mathrm{TP}$ & 120 & 188 & SN & 99 [95-100] & & & & 99 [97-100] & & & & $1.00^{*}$ \\
\hline & $\mathrm{TN}$ & 44 & 75 & SP & & 72 [60-82] & & & & 83 [74-90] & & & 0.10 \\
\hline & FP & 17 & 15 & PPV & & & 88 [81-92] & & & & 93 [88-96] & & 0.12 \\
\hline & $\mathrm{FN}$ & 1 & 1 & NP & & & & 98 [88-100] & & & & 99 [93-100] & $1.00 *$ \\
\hline \multicolumn{2}{|l|}{$P$ value $(\mathrm{L}-\mathrm{I})$} & & & & $0.42 *$ & 0.46 & 0.23 & $1.00^{*}$ & $1.00^{*}$ & $0.52 *$ & $0.19 *$ & $1.00^{*}$ & \\
\hline
\end{tabular}

The diagnostic accuracy (SN, SP) and clinical utility (PPV, NPV) values are expressed as percentages with [95\% confidence interval]. Estimated PTP $[18]<30 \%$ was defined as low and $30-90 \%$ as belonging to the intermediate risk group. $P$ value L-I (low vs. intermediate risk group) and W$\mathrm{M}$ (women vs. men) estimated using chi-squared test and $\left({ }^{*}\right)$ Fisher's exact test

$T P$ true positive, $T N$ true negative, $F P$ false positive, $F N$ false negative, $S N$ sensitivity, $S P$ specificity, $P P V$ positive predictive value, $N P V$ negative predictive value

referred for ICA but underwent additional CTCA we found that:

- The Duke risk score underestimated the prevalence of angiographically obstructive CAD in women, especially in those at low risk;

- The sensitivity and specificity of CTCA were similar across low and intermediate risk groups in and between women and men;

- The clinical utility of a positive CTCA was moderate, with remaining diagnostic uncertainty in low to intermediate risk women and low risk men requiring additional functional testing;
- The yield of obstructive CAD by ICA was similar in women and men with low and intermediate risk;

- CTCA was highly accurate in excluding the presence of obstructive CAD in women and men at low and intermediate risk.

In our study the overall predicted probability of having obstructive CAD using the Duke risk score was underestimated in women ( $45 \%$ vs. $61 \%, P<0.01$ ) compared with the actual presence of CAD assessed by ICA. This finding probably reflects the difficulties of evaluating women with symptoms suspected of CAD and has been described in previous studies [3, 8]. Physicians may misclassify the

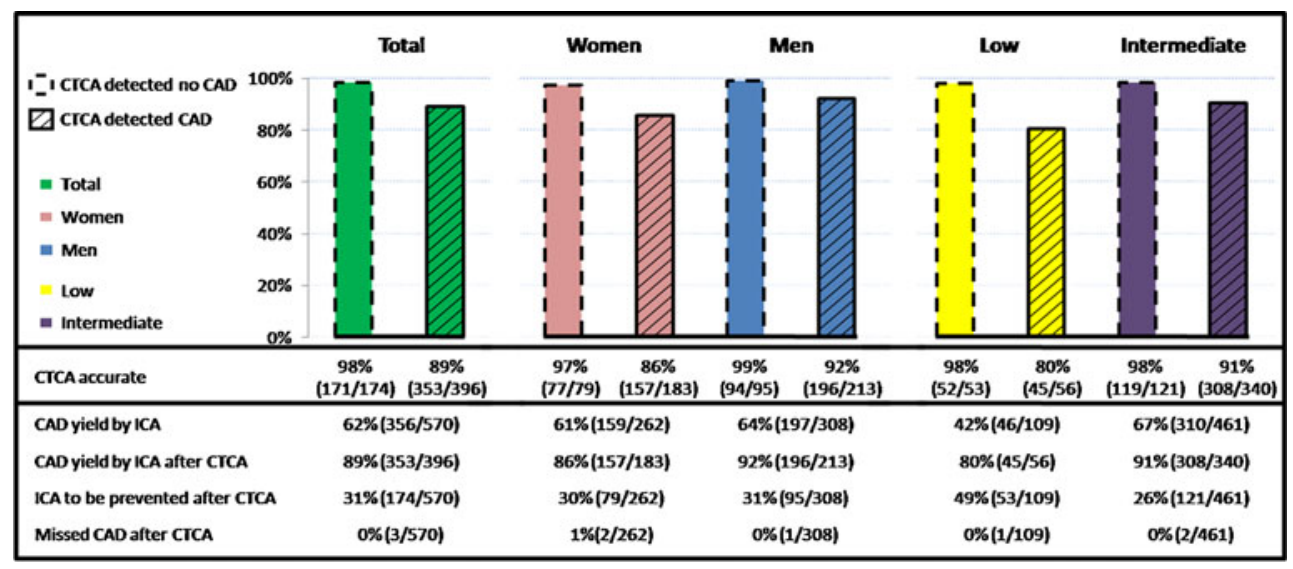

Fig. 1 CAD detection by CTCA and its influence on the yield of CAD by ICA 
symptoms as non-anginal or atypical angina in women, which consequently affects the Duke risk score where symptom presentation plays a significant role in the calculation of the PTP. Underestimation of the Duke risk score may also be related to the referral bias of our study population, in particular in women who were referred by their treating physicians for ICA.

It is noteworthy that the diagnostic accuracy of CTCA in detecting obstructive CAD was similar in women and men (Table 3; sensitivity $99 \%$ vs. $99 \%$; specificity $77 \%$ vs. $85 \%$ for women and men, respectively). Similar findings were shown by Meijboom et al. in a multicentre study (sensitivity $100 \%$ vs. $99 \%$; specificity $63 \%$ vs. $66 \%$ ) [14] and by Pundziute et al. in a single-centre study (sensitivity $95 \%$ vs. $100 \%$; specificity $93 \%$ vs. $89 \%$ for women and men, respectively) [29]. This is in contrast to the reported technical limitations and diminished accuracy in women of other noninvasive ischaemia tests $[6,30]$ and underscores the gender neutrality (equality) of the diagnostic accuracy of CTCA.

The clinical utility of CTCA, i.e. reflecting the remaining diagnostic certainty of the presence or absence of significant $\mathrm{CAD}$, is an important criterion in the overall clinical assessment of CTCA. The clinical utility is derived from the posttest probability of CAD and even with the same diagnostic accuracy of a test (sensitivity/specificity) it is different in patients with low or intermediate PTP of CAD. We arbitrarily assumed that in patients with a post-test probability of less than $5 \%$ or greater than $90 \%$ sufficient diagnostic certainty was achieved and no further diagnostic testing was required. In patients with a post-test probability between $5 \%$ and $90 \%$ diagnostic certainty was not sufficient and in these patients further diagnostic testing was deemed necessary. In our population we found that the clinical utility of a positive CTCA was high ( $>90 \%)$ in men at intermediate PTP of CAD and further diagnostic testing was not necessary. These patients may then be referred to ICA. The clinical utility of a positive CTCA was moderate in women at low to intermediate PTP and in men of low PTP of CAD. In these patients diagnostic certainty was insufficient and further diagnostic testing may be needed. Higher certainty may be then achieved by functional testing for ischaemia e.g. by SPECT or by stress echocardiography $[31,32]$.

The clinical utility of a negative CTCA was high in women and men at low or intermediate PTP of CAD providing high certainty $(<5 \%)$ of absence of obstructive $\mathrm{CAD}$, and no further testing would be necessary. It has been shown in numerous studies that the absence of CAD detected by CTCA is associated with an excellent prognosis [33, 34], which further lends support that these patients may be safely discharged. It should be noted that in patients with persistent chest complaints due to microvascular dysfunction, normal CT findings with no obstructive epicardial CAD may be seen [35]. In these patients, who are often women, further diagnostic testing for ischaemia to detect microvascular dysfunction will be needed to adjust clinical management and survival [36].

Recent studies reported that the yield of elective ICA to demonstrate the presence of obstructive CAD in women and men is $27-49 \%$ and $47-67 \%$, respectively, in patients referred for ICA $[9,10]$, whereas by performing CTCA before diagnostic ICA the yield may improve to $86 \%$ for women and $92 \%$ for men (Fig. 1) and may decrease the number of unnecessary ICA in women and men by $30 \%$ and $31 \%$, respectively, as suggested by our study.

Our study has limitations. Referral bias was present, because the patients in our population were referred by their treating physicians to undergo invasive coronary imaging on the basis of their chest pain presentation with or without outcome of stress testing. This may explain the relatively high prevalence of obstructive $\mathrm{CAD}$, in particular in women. To account for the influence of referral bias on the diagnostic accuracy $[25,26]$ of CTCA we also reported the AUC which was not different between women and men ( 0.87 vs. $0.92, P=$ 0.056 ). Our study population only consisted of stable patients and our results may not apply to the wider spectrum of patients with suspected CAD who did not undergo ICA or who have unstable symptoms. Of concern was the rather high radiation exposure of CTCA $(12 \mathrm{mSv})$ due to the use of a retrospective imaging protocol, which was standard in first-generation CT $[14,37]$. Currently with the newer-generation systems and optimal imaging protocols much lower levels of radiation exposure $(<3 \mathrm{mSv})$ can be achieved in patients with low heart rates $(<65$ beats per minute) [38].

In conclusion, computed tomography coronary angiography has similar diagnostic accuracy in women and men with low and intermediate risk, and may function as an efficient gatekeeper for ICA in women as well as in men.

Acknowledgement All authors contributed substantially to this manuscript and they have read and approved submission of the manuscript.

Funding sources None.

\section{Disclosures None.}

Open Access This article is distributed under the terms of the Creative Commons Attribution License which permits any use, distribution, and reproduction in any medium, provided the original author(s) and the source are credited.

\section{References}

1. Thom T, Haase N, Rosamond W et al (2006) Heart disease and stroke statistics-2006 update: a report from the American Heart Association Statistics Committee and Stroke Statistics Subcommittee. Circulation 113:e85-151 
2. Gholizadeh L, Davidson P (2008) More similarities than differences: an international comparison of CVD mortality and risk factors in women. Health Care Women Int 29:3-22

3. Canto JG, Goldberg RJ, Hand MM et al (2007) Symptom presentation of women with acute coronary syndromes: myth vs reality. Arch Intern Med 167:2405-2413

4. Shaw LJ, Bairey Merz CN, Pepine CJ et al (2006) Insights from the NHLBI-Sponsored Women's Ischemia Syndrome Evaluation (WISE) Study: Part I: gender differences in traditional and novel risk factors, symptom evaluation, and gender-optimized diagnostic strategies. J Am Coll Cardiol 47:S4-S20

5. Detry JM, Kapita BM, Cosyns J, Sottiaux B, Brasseur LA, Rousseau MF (1977) Diagnostic value of history and maximal exercise electrocardiography in men and women suspected of coronary heart disease. Circulation 56:756-761

6. Kwok Y, Kim C, Grady D, Segal M, Redberg R (1999) Metaanalysis of exercise testing to detect coronary artery disease in women. Am J Cardiol 83:660-666

7. Sekhri N, Timmis A, Chen R et al (2008) Inequity of access to investigation and effect on clinical outcomes: prognostic study of coronary angiography for suspected stable angina pectoris. BMJ 336:1058-1061

8. Johnson BD, Kelsey SF, Bairey Merz CN (2003) Clinical risk assessment in women: chest discomfort. In: Shaw LJ, Redberg RF (eds) Coronary disease in women: evidence-based diagnosis and treatment. Humana, Totowa, pp 129-143

9. Patel MR, Peterson ED, Dai D et al (2010) Low diagnostic yield of elective coronary angiography. N Engl J Med 362:886-895

10. Shaw LJ, Shaw RE, Merz CN et al (2008) Impact of ethnicity and gender differences on angiographic coronary artery disease prevalence and in-hospital mortality in the American College of Cardiology-National Cardiovascular Data Registry. Circulation 117:17871801

11. Maas AH, van der Schouw YT, Regitz-Zagrosek V et al (2011) Red alert for women's heart: the urgent need for more research and knowledge on cardiovascular disease in women: proceedings of the workshop held in Brussels on gender differences in cardiovascular disease, 29 September 2010. Eur Heart J 32:1362-1368

12. Chow BJ, Abraham A, Wells GA et al (2009) Diagnostic accuracy and impact of computed tomographic coronary angiography on utilization of invasive coronary angiography. Circ Cardiovasc Imaging 2:16-23

13. Budoff MJ, Dowe D, Jollis JG et al (2008) Diagnostic performance of 64-multidetector row coronary computed tomographic angiography for evaluation of coronary artery stenosis in individuals without known coronary artery disease: results from the prospective multicenter ACCURACY (Assessment by Coronary Computed Tomographic Angiography of Individuals Undergoing Invasive Coronary Angiography) trial. J Am Coll Cardiol 52:1724-1732

14. Meijboom WB, Meijs MF, Schuijf JD et al (2008) Diagnostic accuracy of 64-slice computed tomography coronary angiography: a prospective, multicenter, multivendor study. J Am Coll Cardiol 52:2135-2144

15. Mowatt G, Cummins E, Waugh N et al (2008) Systematic review of the clinical effectiveness and cost-effectiveness of 64-slice or higher computed tomography angiography as an alternative to invasive coronary angiography in the investigation of coronary artery disease. Health Technol Assess 12:iii-iv, ix-143

16. Meijboom WB, Weustink AC, Pugliese F et al (2007) Comparison of diagnostic accuracy of 64-slice computed tomography coronary angiography in women versus men with angina pectoris. Am J Cardiol 100:1532-1537

17. Miller JM, Rochitte CE, Dewey M et al (2008) Diagnostic performance of coronary angiography by 64-row CT. N Engl J Med 359:2324-2336

18. Pryor DB, Shaw L, McCants CB et al (1993) Value of the history and physical in identifying patients at increased risk for coronary artery disease. Ann Intern Med 118:81-90
19. Agatston AS, Janowitz WR, Hildner FJ, Zusmer NR, Viamonte M Jr, Detrano R (1990) Quantification of coronary artery calcium using ultrafast computed tomography. J Am Coll Cardiol 15:827832

20. Austen WG, Edwards JE, Frye RL et al (1975) A reporting system on patients evaluated for coronary artery disease. Report of the Ad Hoc Committee for Grading of Coronary Artery Disease, Council on Cardiovascular Surgery, American Heart Association. Circulation 51:5-40

21. Skinner JS, Smeeth L, Kendall JM, Adams PC, Timmis A, Chest Pain Guideline Development Group (2010) NICE guidance. Chest pain of recent onset: assessment and diagnosis of recent onset chest pain or discomfort of suspected cardiac origin. Heart 96:974-978

22. Meijboom WB, van Mieghem CA, Mollet NR et al (2007) 64-slice computed tomography coronary angiography in patients with high, intermediate, or low pretest probability of significant coronary artery disease. J Am Coll Cardiol 50:1469-1475

23. Dharampal AS, Rossi A, Papadopoulou SL et al (2011) Is there a difference in the diagnostic accuracy of computed tomography coronary angiography between women and men? Coron Artery Dis 22:421-427

24. Wilson EB (1927) Probable inference, law of succession, and statistical inference. J Am Stat Assoc 22:209-212

25. Diamond GA (1991) Affirmative actions: can the discriminant accuracy of a test be determined in the face of selection bias? Med Decis Making 11:48-56

26. Fischer JE, Bachmann LM, Jaeschke R (2003) A readers' guide to the interpretation of diagnostic test properties: clinical example of sepsis. Intensive Care Med 29:1043-1051

27. Weustink AC, Mollet NR, Neefjes LA et al (2010) Diagnostic accuracy and clinical utility of noninvasive testing for coronary artery disease. Ann Intern Med 152:630-639

28. Bossuyt PM, Reitsma JB, Bruns DE et al (2003) Towards complete and accurate reporting of studies of diagnostic accuracy: the STARD initiative. Clin Radiol 58:575-580

29. Pundziute G, Schuijf JD, Jukema JW et al (2008) Gender influence on the diagnostic accuracy of 64-slice multislice computed tomography coronary angiography for detection of obstructive coronary artery disease. Heart 94:48-52

30. Grady D, Chaput L, Kristof M (2003) Diagnosis and treatment of coronary heart disease in women: systematic reviews of evidence on selected topics. Evidence report/technology assessment 81. Agency for Healthcare Research and Quality, Rockville

31. Wijns W, Kolh P, Danchin N et al (2010) Guidelines on myocardial revascularization. Eur Heart J 31:2501-2555

32. Anderson JL, Adams CD, Antman EM et al (2011) 2011 ACCF/ AHA focused update incorporated into the ACC/AHA 2007 guidelines for the management of patients with unstable angina/non-STelevation myocardial infarction: a report of the American College of Cardiology Foundation/American Heart Association Task Force on Practice guidelines. Circulation 123:e426-579

33. Min JK, Dunning A, Lin FY et al (2011) Age- and sex-related differences in all-cause mortality risk based on coronary computed tomography angiography findings results from the International Multicenter CONFIRM (Coronary CT Angiography Evaluation for Clinical Outcomes: an International Multicenter Registry) of 23,854 patients without known coronary artery disease. J Am Coll Cardiol 58:849-860

34. Ostrom MP, Gopal A, Ahmadi N et al (2008) Mortality incidence and the severity of coronary atherosclerosis assessed by computed tomography angiography. J Am Coll Cardiol 52:1335-1343

35. Johnson BD, Shaw LJ, Pepine CJ et al (2006) Persistent chest pain predicts cardiovascular events in women without obstructive coronary artery disease: results from the NIH-NHLBI-sponsored Women's Ischaemia Syndrome Evaluation (WISE) study. Eur Heart J 27:14081415 
36. Reis SE, Holubkov R, Conrad Smith AJ et al (2001) Coronary microvascular dysfunction is highly prevalent in women with chest pain in the absence of coronary artery disease: results from the NHLBI WISE study. Am Heart J 141:735-741

37. Weustink AC, Mollet NR, Pugliese F et al (2008) Optimal electrocardiographic pulsing windows and heart rate: effect on image quality and radiation exposure at dual-source coronary CT angiography. Radiology 248:792-798

38. Neefjes LA, Dharampal AS, Rossi A et al (2011) Image quality and radiation exposure using different low-dose scan protocols in dual-source CT coronary angiography: randomized study. Radiology 261:779-786 